\title{
Nab: Measurement Principles, Apparatus and Uncertainties
}

\author{
Dinko Počanić $^{\mathrm{a}, *}$, R. Alarcon ${ }^{\mathrm{b}}$, L.P. Alonzi ${ }^{\mathrm{a}}$, S. Baeßler ${ }^{\mathrm{a}}$, \\ S. Balascuta ${ }^{b}$, J.D. Bowman ${ }^{h}$, M.A. Bychkov ${ }^{a}$, J. Byrne $^{j}$,

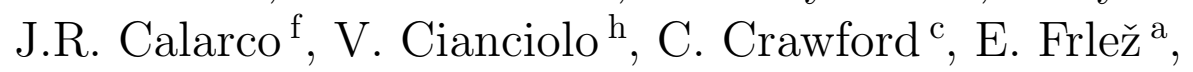 \\ M.T. Gericke ${ }^{\mathrm{d}}$, G.L. Greene ${ }^{\mathrm{k}}$, R.K. Grzywacz ${ }^{\mathrm{k}}$, V. Gudkov ${ }^{\mathrm{i}}$, \\ F.W. Hersman ${ }^{\text {f }}$, A. Klein ${ }^{\mathrm{e}}$, J. Martin ${ }^{\ell}$, S.A. Page ${ }^{\mathrm{d}}$, \\ A. Palladino ${ }^{\text {a }}$, S.I. Penttilä ${ }^{\text {h}}$, K.P. Rykaczewski ${ }^{\mathrm{h}}$, \\ W.S. Wilburn ${ }^{\mathrm{e}}$, A.R. Young ${ }^{\mathrm{g}}$, G.R. Young ${ }^{\mathrm{h}}$ \\ The Nab collaboration

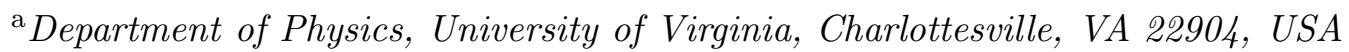 \\ ${ }^{\mathrm{b}}$ Department of Physics, Arizona State University, Tempe, AZ 85287-1504, USA \\ ${ }^{\mathrm{c}}$ Department of Physics and Astronomy, University of Kentucky, Lexington, \\ Kentucky 40506, USA \\ ${ }^{\mathrm{d}}$ Department of Physics and Astronomy, University of Manitoba, Winnipeg, \\ Manitoba, R3T 2N2 Canada \\ ${ }^{\mathrm{e}}$ Los Alamos National Laboratory, Los Alamos, NM 87545, USA \\ ${ }^{\mathrm{f}}$ Department of Physics, University of New Hampshire, Durham, NH 03824, USA \\ ${ }^{\mathrm{g}}$ Department of Physics, North Carolina State University, Raleigh, NC \\ 27695-8202, USA \\ ${ }^{\mathrm{h}}$ Physics Division, Oak Ridge National Laboratory, Oak Ridge, TN 37831, USA \\ ${ }^{\mathrm{i}}$ Department of Physics and Astronomy, University of South Carolina, Columbia, \\ SC 29208, USA \\ ${ }^{\mathrm{j} D e p a r t m e n t}$ of Physics and Astronomy, University of Sussex, Brighton, BN19RH, \\ $U K$ \\ ${ }^{\mathrm{k}}$ Department of Physics and Astronomy, University of Tennessee, Knoxville, TN \\ 37996-1200, USA \\ ${ }^{\ell}$ Department of Physics, University of Winnipeg, Winnipeg, Manitoba, R3B 2E9 \\ Canada
}

Preprint submitted to Elsevier

30 September 2008 


\begin{abstract}
The Nab collaboration will perform a precise measurement of $a$, the electronneutrino correlation parameter, and $b$, the Fierz interference term in neutron beta decay, in the Fundamental Neutron Physics Beamline at the SNS, using a novel electric/magnetic field spectrometer and detector design. The experiment is aiming at the $10^{-3}$ accuracy level in $\Delta a / a$, and will provide an independent measurement of $\lambda=G_{A} / G_{V}$, the ratio of axial-vector to vector coupling constants of the nucleon. Nab also plans to perform the first ever measurement of $b$ in neutron decay, which will provide an independent limit on the tensor weak coupling.
\end{abstract}

Key words: neutron beta decay, correlations, precision measurement PACS: 13.30.-Ce, 14.20.Dh, 23.40.-s, 24.80.-y

\title{
1 Motivation
}

Neutron beta decay provides one of the most sensitive means for exploring details and limits of our understanding of the weak interaction. Thanks to its highly precise theoretical description [1, neutron decay is sensitive to contributions from processes not included in the standard model (SM) of particles and interactions (for comprehensive reviews see Refs. [2,3,4]). Neglecting recoil, radiative and loop corrections, the differential decay rate for unpolarized neutrons is given by parameters $a$ and $b: d w \propto 1+a \beta_{\mathrm{e}} \cos \theta_{\mathrm{e} \nu}+b\left(m_{\mathrm{e}} / E_{\mathrm{e}}\right)$, where $\beta_{\mathrm{e}}=p_{\mathrm{e}} / E_{\mathrm{e}}, p_{\mathrm{e}}, E_{\mathrm{e}}$ and $\theta_{e \nu}$ are the electron momentum, energy, and $\mathrm{e}-\nu$ opening angle, respectively [5]. The $\mathrm{e}-\nu_{\mathrm{e}}$ correlation parameter $a$, and the asymmetry parameters with respect to the neutron spin: $A$ (beta), $B$ (neutrino), and $C$ (proton; $C \propto A+B$ in leading order) possess complementary dependencies on the ratio of Fermi constants $\lambda=G_{A} / G_{V}$, as well as on operators that depart from the $(V-A) \otimes(V-A)$ form of the SM charged current (CC) weak interaction. Additionally, $b$, the Fierz interference term, offers an independent test of scalar and tensor admixtures arising in broad classes of L-R mixing SUSY extensions. Thus precise measurements of neutron decay parameters offer the distinct advantage of overconstrained independent checks of the SM predictions, as well as the potential for indicating or ruling out certain types of extensions to the $\mathrm{SM}(V-A) \otimes(V-A)$ form [2,3, 4,6$]$. Hence, a set of appropriately precise measurements of the neutron decay parameters $a, b, A$, and $B$ will have considerably greater physics implications than the erstwhile predominant experimental focus on $A$, i.e., $\lambda$. At a minimum,

\footnotetext{
* Corresponding author: pocanic@virginia.edu
} 
such a data set combined with new measurements of the neutron lifetime, $\tau_{\mathrm{n}}$, will enable a definitive resolution of the persistent discrepancies in $\lambda$ and Cabibbo-Kobayashi-Maskawa (CKM) matrix element $V_{u d}$ [7].

The Nab collaboration [8] has undertaken to carry out precise measurements of $a$, the e $-\nu_{\mathrm{e}}$ correlation parameter, and $b$, the so far unmeasured Fierz interference term, in neutron decay. Goal accuracies are $\Delta a / a \simeq 10^{-3}$ and $\Delta b \simeq 10^{-3}$. A novel $4 \pi$ field-expansion spectrometer based on ideas outlined in Ref. [11] will be used in the Fundamental Neutron Physics Beamline (FnPB) at the Spallation Neutron Source (SNS) at Oak Ridge, Tennessee.

The Nab experiment constitutes the first phase of a program of measurements that will continue with second-generation measurements of spin correlations in neutron decay. The next experiment, named 'abBA', will measure parameters $A$ and $B$ in addition to $a$ and $b$. In addition, the proton asymmetry $C$ will be measured with the same apparatus. Together, Nab and abBA form a complete program of measurements of the main neutron decay parameters in a single apparatus with shared systematics and consistency checks. The experiments are complementary: Nab is highly optimized for the measurement of $a$ and $b$, while abBA focuses on $A$ and $B$ with a lower-precision consistency check of the $a$ and $b$ parameters. Nab joins two existing experiments, aSPECT [9] and aCORN [10], which also study $a$.

\section{Measurement Principles and Apparatus}

The correlation parameter of interest, $a$, measures the dependence of the neutron beta decay rate on the cosine of the e- $\nu$ relative angle. The Nab method of determination of $a$ relies on the linear dependence of $\cos \theta_{\mathrm{e} \nu}$ on $p_{\mathrm{p}}^{2}$, the square of the proton momentum for a given electron momentum (or energy). Conservation of momentum gives the relation

$$
p_{\mathrm{p}}^{2}=p_{\mathrm{e}}^{2}+2 p_{\mathrm{e}} p_{\nu} \cos \theta_{\mathrm{e} \nu}+p_{\nu}^{2}
$$

where, to a very good approximation, $p_{\nu}$ depends only on $E_{\mathrm{e}}$ (or $p_{\mathrm{e}}$ ). Thus, Eq. 1 reduces to a linear relation between $\cos \theta_{\mathrm{e} \nu}$ and $p_{\mathrm{p}}^{2}$ for a fixed $p_{\mathrm{e}}$. The mapping of $\cos \theta_{\mathrm{e} \nu}$ and $p_{\mathrm{p}}^{2}$ is shown graphically in Fig. 1. In this plot, the phase space alone distributes proton events evenly in $p_{\mathrm{p}}^{2}$ between the lower and upper bounds for any fixed value of $E_{e}$. Given the linear relationship between $p_{\mathrm{p}}^{2}$ and $\cos \theta_{\mathrm{e} \nu}$, the slope of the $p_{\mathrm{p}}^{2}$ probability distribution is determined by the correlation parameter $a$; in fact it is given by $\beta a$, where $\beta=v_{\mathrm{e}} / c$ (see Fig. 2). This observation leads to the main principle of measurement of $a$ which involves measurement of the proton momenta via the proton time of flight $(\mathrm{TOF}), t_{\mathrm{p}}$, in a suitably constructed magnetic spectrometer. Ideally, the 
magnetic field longitudinalizes the proton momentum and $t_{\mathrm{p}} \propto 1 / p_{\mathrm{p}} ; t_{\mathrm{p}}$ is measured as the difference between the arrival times of the electron and the proton at the detector(s). In the present discussion we neglect the electron TOF. Parameter $a$ is determined from the slopes of the $1 / t_{\mathrm{p}}^{2}$ distributions for different values of $E_{\mathrm{e}}$. If $a$ were null, all distributions would have a slope of zero. Having multiple independent measurements of $a$ for different electron energies provides a powerful check of systematics, as discussed below. The Fierz interference term $b$ is determined from the shape of the measured electron energy spectrum.

For fixed $E_{\mathrm{e}}$, a perfect spectrometer would record a trapezoidal distribution of $1 / t_{\mathrm{p}}^{2}$ with sharp edges. The precise location of these edges is determined by well-defined kinematic cutoffs that only depend on $E_{\mathrm{e}}$. However, a realistic time-of-flight spectrometer will produce imperfect measurements of the proton momenta due to the spectrometer response function, discussed in Sect. 3. The measured locations and shapes of edges in $1 / t_{\mathrm{p}}^{2}$ distributions will allow us to examine the spectrometer response function and verify that the fields have been measured correctly.

The main requirements on the spectrometer are:

(1) The spectrometer and its magnetic $(\vec{B})$ and electric $(\vec{E})$ fields are de-

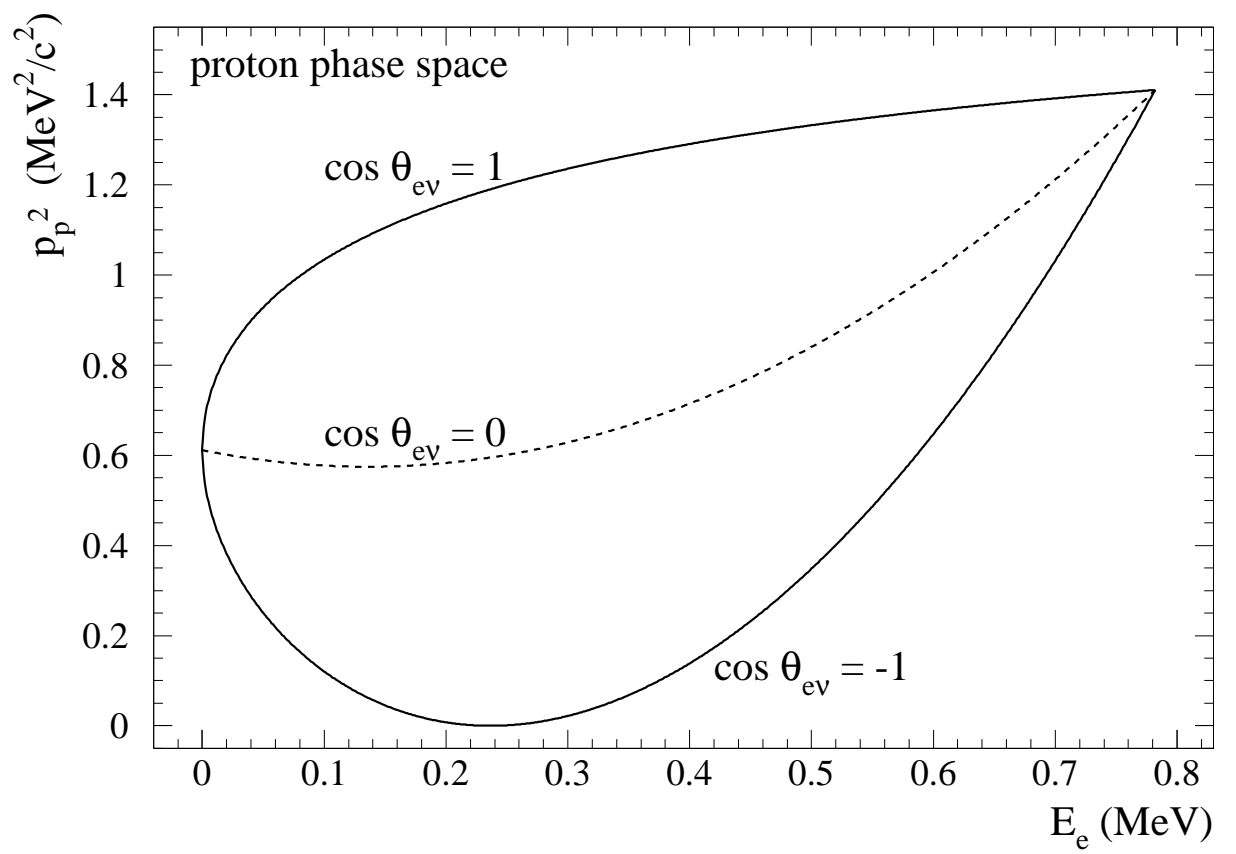

Fig. 1. Proton phase space (in terms of $p_{\mathrm{p}}^{2}$ ) in neutron beta decay as a function of electron kinetic energy. The upper bound of the allowed phase space occurs for collinear e and $\nu$ momenta, $\cos \theta_{\mathrm{e} \nu}=1$, while the momenta are anticollinear, $\cos \theta_{\mathrm{e} \nu}=-1$, at the lower bound. The central dashed parabola corresponds to orthogonal $e$ and $\nu$ momenta. 


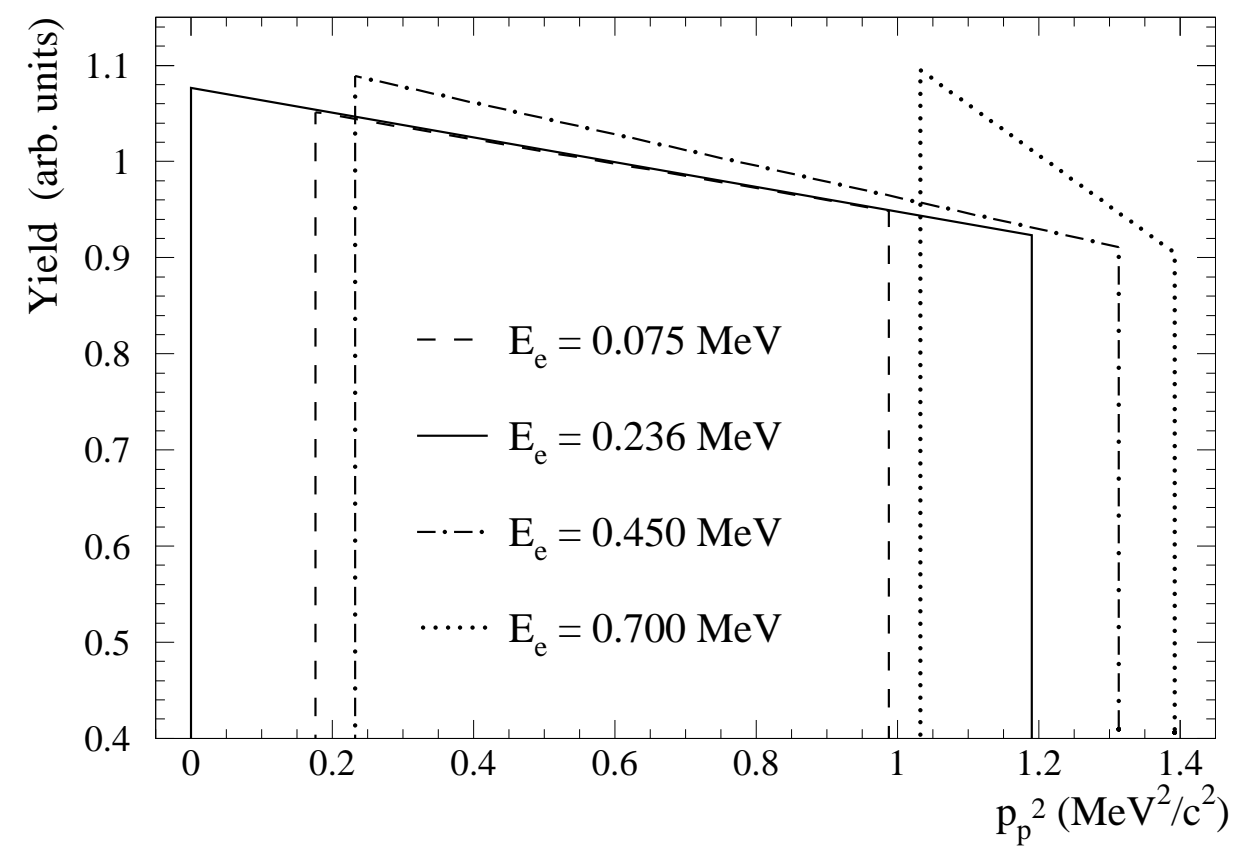

Fig. 2. A plot of proton yield for four different electron kinetic energies with $a=-0.105$. If $a$ were 0 , all the distributions would have a slope of 0 . Vertical scale origin is suppressed.

signed to be azimuthally symmetric about the central axis, $z$.

(2) Neutrons must decay in a region of large $\vec{B}$. The resulting protons and electrons spiral around a magnetic field line.

(3) An electric field is required to accelerate the proton from the eV-range energies to a detectable energy range prior to reaching the detector. This field imposes, however, an energy threshold on $\mathrm{e}^{-}$detection.

(4) The proton momentum must rapidly become parallel to the magnetic field direction to ensure that the proton time of flight $t_{\mathrm{p}} \propto 1 / p_{\mathrm{p}}$. This requirement dictates a sharp field curvature $\left(d^{2} B_{z} / d z^{2}\right)$ at the origin, followed by a sharp falloff of $B_{z}$.

The basic concept of the spectrometer consists of collinear solenoids with their longitudinal axis oriented normal to the neutron beam, which passes through the solenoid center. The solenoidal magnetic field starts out high at the position of the neutron beam, typically $4 \mathrm{~T}$, dropping off quickly to parallelize the momenta as protons enter the long "drift" region. In the detection region at either end of the solenoid the field is increased to $1 / 4$ of its central peak value. Cylindrical electrodes (consisting of three sections) maintain the neutron decay region at a potential of $+30 \mathrm{kV}$ with respect to the ends of the solenoid where detectors are placed at ground potential.

The magnetic field strength is sufficiently high to constrain both electrons and protons from neutron decay to spiral along the magnetic field lines with the component of the spiral motion transverse to the field limited by cyclotron 

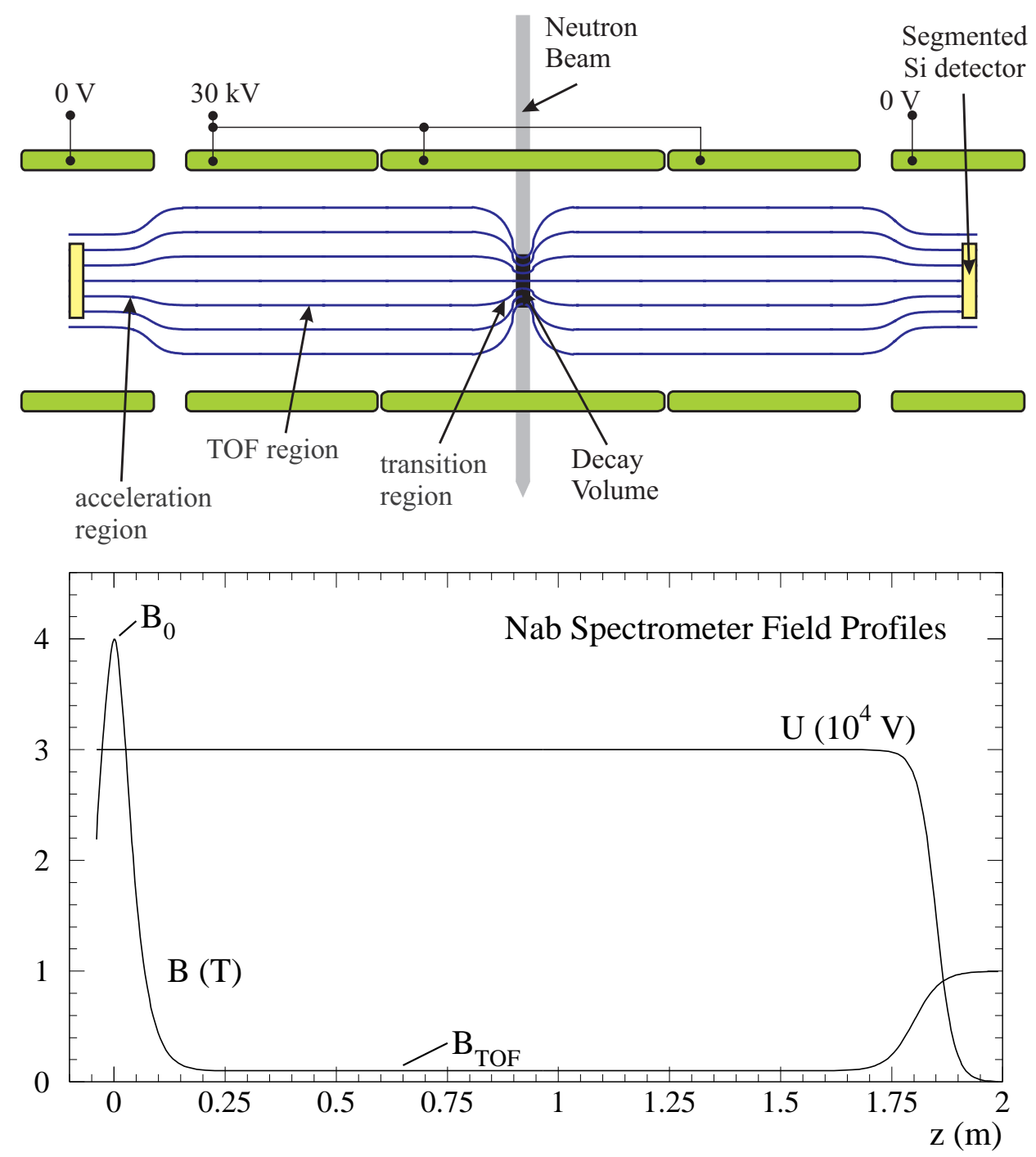

Fig. 3. Top panel: A schematic view of the vertical field expansion spectrometer showing the main regions of the device: (a) neutron decay region, (b) transition region with expanding magnetic field, (c) drift (TOF) region, and (d) the acceleration region before the detector. Bottom panel: Electrical potential $(U)$ and magnetic field $(B)$ profiles on axis for $1 / 2$ of the Nab spectrometer length.

radii of the order of a few millimeters.

Hence, two segmented Si detectors, one at each end of the solenoid, view both electrons and protons in an effective $4 \pi$ geometry. The time of flight between the electron and proton is accurately measured in a long, $l \sim 1.5$ meter, drift distance. The electron energy is accurately measured in the Si detectors. The proton momentum and electron energy determine the electron-neutrino opening angle. We note that by sorting the data on proton time of flight and electron energy, $a$ can be determined with a statistical uncertainty that is only $4 \%$ greater than the theoretical minimum [14]. 
A not-to-scale schematic view of the field expansion spectrometer is shown in Fig. 3, Electrons and protons spiral around magnetic field lines and are guided to two segmented $\mathrm{Si}$ detectors, each having a $\sim 100 \mathrm{~cm}^{2}$ active area, and depicted schematically in Fig. 4. In the center of the spectrometer the axial field strength is $4 \mathrm{~T}$, in the drift region $0.1 \mathrm{~T}$, and near the Si detectors $1 \mathrm{~T}$ (see Fig. 3).

In a realistic spectrometer, however, the perfect one-to-one correspondence of proton momentum and time of flight is lost, due to imperfect momentum longitudinalization and other systematic effects, such as the lateral size of the neutron beam in the decay region. In other words, the detector response function instead of being a delta function in $1 / t_{\mathrm{p}}^{2}$ for each value of $p_{\mathrm{p}}^{2}$, becomes a broadened function, such as the ones calculated for three proton momenta and depicted in Fig. 5. The key challenge of the Nab approach to measuring $a$ is to minimize the width of the detector response function while keeping the relevant systematics under control. The resulting TOF distributions no longer have sharply cut off edges as in Fig. 2. A sample set of results of GEANT4 [12] Monte Carlo calculations for three electron energies is shown in Fig. 6.

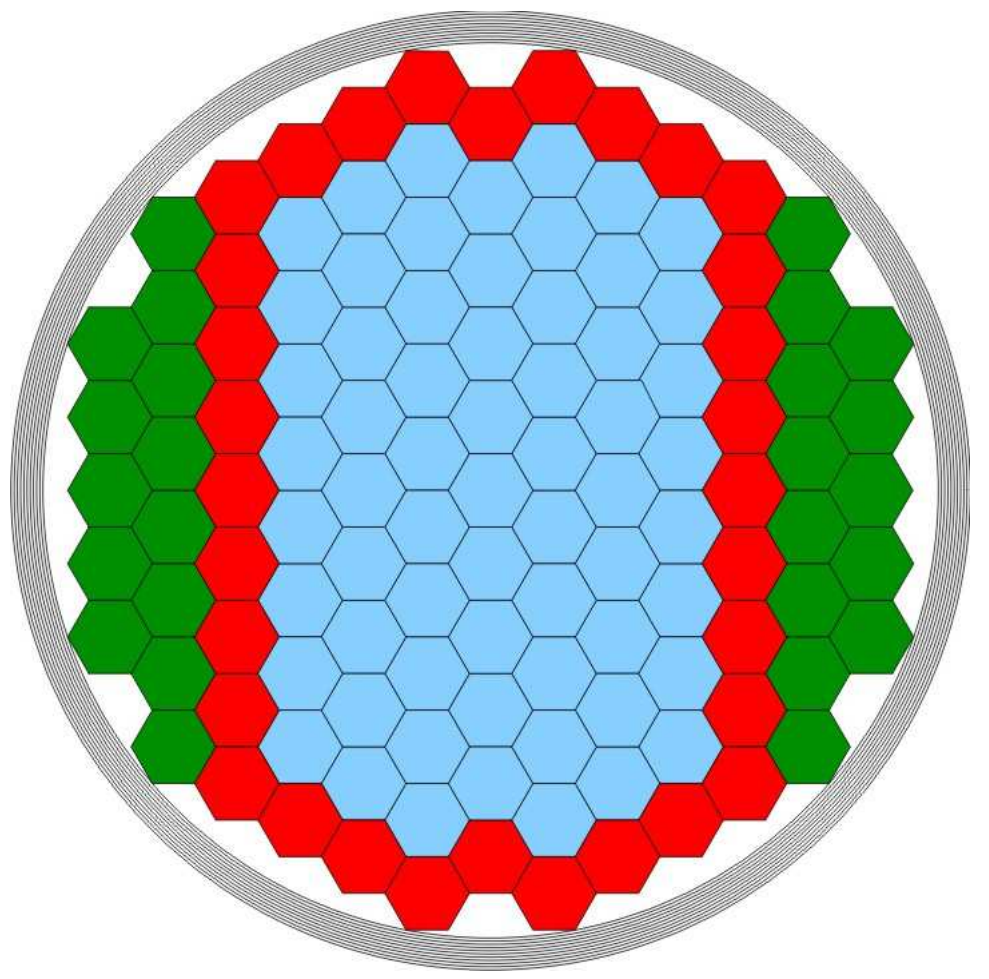

Fig. 4. Design for the ohmic side of the detector. The 127 hexagons represent individual detector elements. Proton events in the interior hexagons generate a valid trigger, while the perimeter hexagons are used only for detecting electrons. The concentric circles represent the guard ring structure. Electrical contact is made to each hexagon to provide the bias voltage and collect the charge deposited by incident particles. The areas between the pixels and guard rings are electrically connected to form one additional channel. 


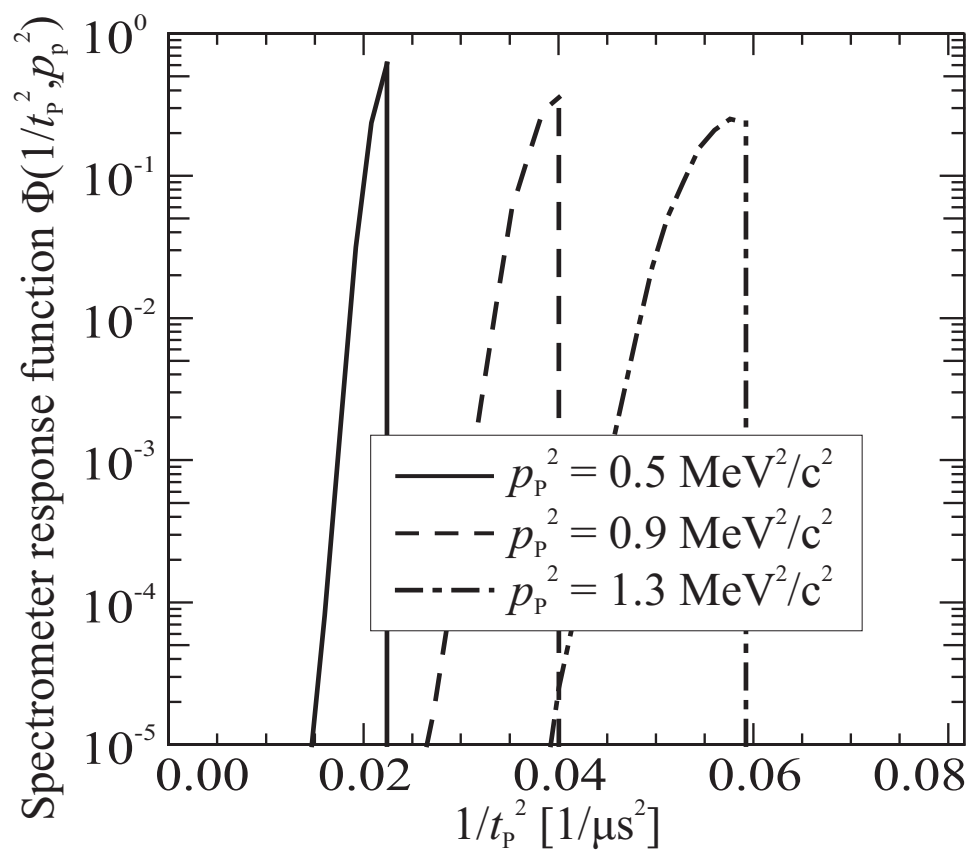

Fig. 5. Nab spectrometer response function $\Phi$, shown for different proton momenta, the magnetic field from Fig. 3 and a centered neutron beam with a width of $2 \mathrm{~cm}$. The calculation assumes full adiabaticity of the proton motion.

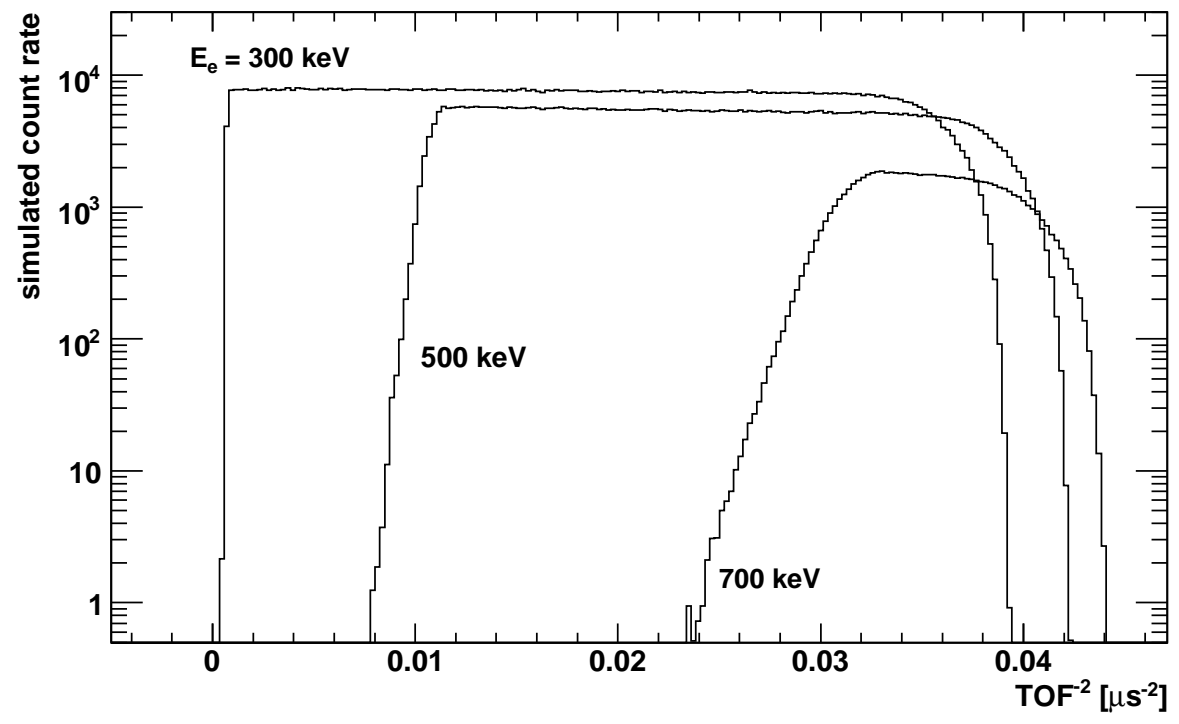

Fig. 6. Proton TOF spectra, $P_{t}\left(1 / t_{\mathrm{p}}^{2}\right)$, for electron kinetic energies $E_{\mathrm{e}}=300,500$ and $700 \mathrm{keV}$, generated in a realistic GEANT4 Monte-Carlo simulation using the $\vec{B}$ field from Fig. 3 and a centered neutron beam with a width of $2 \mathrm{~cm}$.

Strictly speaking, determining $b$ requires detecting only the electron and reliably measuring its kinetic energy. Nevertheless, there are a number of challenges associated with this measurement, commented on in the following Section. 


\section{Measurement Uncertainties and Systematics}

The statistical sensitivity of our measurement method is primarily determined by the spectrometer acceptance and imposed energy and TOF restrictions. The statistical uncertainties for our measurements of the $a$ and $b$ parameters in neutron decay are listed in Tab. 1, reflecting the dependence on $E_{\mathrm{e}, \min }$, the electron kinetic energy detection threshold, and $t_{\mathrm{p}, \max }$, the maximum proton TOF accepted. Additionally, the electron energy calibration $E_{\text {cal }}$ and the precise length $l$ of the low-field drift region represent important sources of systematic uncertainty. Thus, parallel analyses will be performed keeping $E_{\text {cal }}$ and $l$ free, in order to study and remove their systematic effects. Table 1 shows that the reduction in statistical sensitivities under these conditions is modest.

The calculated FnPB neutron decay rate under SNS full-power conditions of $\sim 19.5 /\left(\mathrm{cm}^{3} \mathrm{~s}\right)$, and with the Nab fiducial decay volume of $20 \mathrm{~cm}^{3}$, yields $\sim 400$ detected decays/sec [13]. In a typical 10-day run of $7 \times 10^{5} \mathrm{~s}$ of net beam time we would achieve $\sigma_{a} / a \simeq 2 \times 10^{-3}$ and $\sigma_{b} \simeq 6 \times 10^{-4}$. Since we plan to collect several samples of $10^{9}$ events in several 6 -week runs, the overall Nab accuracy will not be statistics-limited.

Controlling the measurement systematics presents by far the greatest challenge in the Nab experiment. The most basic task is to specify the spectrometer fields with precision sufficient for an accurate determination of the spectrometer response function $\Phi\left(1 / t_{\mathrm{p}}^{2}, p_{\mathrm{p}}^{2}\right)$. We have adopted two methods of addressing this problem. In the first approach (Method A), we determine the shape of the spectrometer response function from theory, leaving several parameters free, to be determined by fits to the measured spectra. The second approach (Method B) relies on obtaining the detection function with its uncertainties

Table 1

Top: statistical uncertainties $\sigma_{a}$ for the e- $\nu$ correlation parameter $a$. A perfect spectrometer would obtain $\sigma_{a}=2.3 / \sqrt{N}$. Bottom: statistical uncertainties $\sigma_{b}$ for the Fierz interference term $b$.

\begin{tabular}{ccccc}
\hline$E_{\mathrm{e}, \min }$ & 0 & $100 \mathrm{keV}$ & $100 \mathrm{keV}$ & $300 \mathrm{keV}$ \\
$t_{\mathrm{p}, \max }$ & - & - & $10 \mu \mathrm{s}$ & $10 \mu \mathrm{s}$ \\
\hline \hline$\sigma_{a}$ & $2.4 / \sqrt{N}$ & $2.5 / \sqrt{N}$ & $2.6 / \sqrt{N}$ & $3.5 / \sqrt{N}$ \\
$\sigma_{a}^{\dagger}$ & $2.5 / \sqrt{N}$ & $2.6 / \sqrt{N}$ & - & - \\
\hline \hline$E_{\mathrm{e}, \min }$ & 0 & $100 \mathrm{keV}$ & $200 \mathrm{keV}$ & $300 \mathrm{keV}$ \\
\hline \hline$\sigma_{b}$ & $7.5 / \sqrt{N}$ & $10.1 / \sqrt{N}$ & $15.6 / \sqrt{N}$ & $26.4 / \sqrt{N}$ \\
$\sigma_{b}^{\dagger \dagger}$ & $9.1 / \sqrt{N}$ & $12.7 / \sqrt{N}$ & $20.3 / \sqrt{N}$ & $35.1 / \sqrt{N}$ \\
\hline
\end{tabular}

${ }^{\dagger}$ with $E_{\text {cal }}$ and $l$ variable. ${ }^{\dagger \dagger}$ with $E_{\text {cal }}$ and $a$ variable. 
a priori from a full description of the neutron beam and electromagnetic field geometry. Subsequently, the experimental data are fitted with only the physics observables as free parameters. Below we summarize some of the main challenges along with strategies for their control at the required level. A much more detailed discussion of both methods and the experimental challenges is given in the Nab experiment proposal [14].

$\underline{\text { Uncertainties in } a \text { due to the spectrometer response }}$

- Precise specification of the neutron beam profile: A mere $100 \mu \mathrm{m}$ shift of the beam center induces $\Delta a / a \sim 0.2 \%$. However, this effect cancels when averaging over the two detectors on opposite sides of the solenoid; measuring a nonzero up-down proton counting asymmetry pins it down sufficiently.

- Magnetic field map: The field expansion ratio defined as $r_{\mathrm{B}}=B_{\mathrm{TOF}} / B_{0}$ must be controlled at the level of $\Delta r_{\mathrm{B}} / r_{\mathrm{B}}=10^{-3}$ in order to keep $\Delta a / a$ under $10^{-3}$. This will be mapped out using a calibrated Hall probe. Field curvature must be determined with an accuracy of $1 \times 10^{-3}$ in dedicated measurements. Average mapping accuracy $\Delta B / B$ must be kept below $\sim 2 \times 10^{-3}$.

- Flight path length: An uncertainty of order $\Delta l \leq 30 \mu \mathrm{m}$ results in $\Delta a / a$ at our limit. Hence, $l$ will be kept as a fitting parameter. Additionally, we will perform a consistency check by making differential measurement using segmented electrodes.

- Homogeneity of the electric field: Electric potential will have satisfy stringent limits on inhomogeneities as discussed in the Nab proposal [14].

- Rest gas: requires vacuum of $10^{-7} \mathrm{~Pa}$ or better.

- Adiabaticity of the magnetic field configuration is not an absolute requirement. Detailed Monte Carlo analysis has shown excellent efficiency of proton momentum longitudinalization for certain relatively non-adiabatic fields. However, an adiabatic design makes the evaluation of systematic errors simpler and more reliable.

- Doppler effect: Adverse effects of the Doppler effect will apparently be controlled sufficiently by the spectrometer design, but a thorough analysis will be made in conjunction with the final design.

\section{$\underline{\text { Uncertainties in a due to the detector }}$}

- Detector alignment: The spectrometer imaging properties provide for a selfconsistent calibration in the data.

- Electron energy calibration is required at the $10^{-4}$ level. To achieve it we'll use radioactive sources, evaluate directional count rate asymmetries, and also leave it as a fitting parameter with acceptably small loss of statistical sensitivity (see Tab. 1).

- Trigger hermiticity is affected by the particle impact angle on the detector, backscattering, and TOF cutoff (planned in order to reduce accidental backgrounds). Several consistency checks will be evaluated from the data to 
quantify and characterize the various aspects of trigger hermiticity.

- TOF measurement uncertainties: The requirement is $\Delta\left(t_{\mathrm{p}}-t_{\mathrm{e}}\right) \sim 100 \mathrm{ps}$. While it is not necessary to reach this timing accuracy for each event, it has to be achieved for the event sample average, a realistic goal given the planned event statistics.

- Edge effects introduce important systematics. Thanks to the imaging properties of the spectrometers, these can be controlled and corrected for to a sufficient degree with appropriate cuts on the data.

\section{Uncertainties in $b$}

Sources of uncertainties in the measurement of $b$ are fewer than for $a$ since accurate proton momentum measurement (via its TOF) is not required. The dominant sources are electron energy calibration (discussed above) and electron backgrounds.

\section{Backgrounds for $a$ and $b$}

- Neutron beam related backgrounds are notoriously hard to calculate and model a priori, and will ultimately have to be measured and characterized in situ. Reasonable estimates place the beam-related background rates below the signal rate. While we have plans for shielding and lining surfaces with neutron absorbing ${ }^{6} \mathrm{LiF}$ material, the coincident technique of detecting ep pairs helps to reduce substantially the effect of beam-related accidental backgrounds.

- Particle trapping: Electrons can be trapped in the decay volume, expansion, and TOF regions. These regions form an electrode-less Penning trap. The potential well trap does not cause a problem for electrons above our energy threshold. The longitudinalization of the electron momentum due to the magnetic field allows all of them to escape and to reach the detector. Low energy electrons from neutron decay, from field ionization or from rest gas interactions are a concern since trapped particles ionize the rest gas, and the ions form a time-dependent background. Several strategies are under consideration to remove the trapped particles; they will be refined under real running conditions.

\section{Summary}

The Nab collaboration plans simultaneous high-statistics measurements of neutron decay parameters $a$, the e- $\nu$ correlation coefficient, and $b$, the Fierz interference term, with $\Delta a / a \simeq 10^{-3}$ and $\Delta b \simeq 3 \times 10^{-3}$.

Basic properties of the Nab spectrometer are well understood; details of the fields are under study in extensive analytical and Monte Carlo calculations. 
Elements of the proposed Nab spectrometer will be shared with other neutron decay experiments, such as abBA.

Development of the abBA/Nab Si detectors is ongoing and remains a technological challenge. Each of the target properties of the detector have been realized separately; the remaining task is to realize them simultaneously in one piece of silicon.

The major elements of the data acquisition system have been successfully developed.

The experiment received approval in Feb. 2008. Under the most favorable funding and technical scenario it could be ready for commissioning in 2010 .

\section{References}

[1] A. Czarnecki, W.J. Marciano, and A. Sirlin, Phys. Rev. D 70 (2004) 093006.

[2] P. Herczeg, Prog. Part. Nucl. Phys. 46 (2001) 413.

[3] N. Severijns, M. Beck, O. Naviliat-Čunčić, Rev. Mod. Phys. 78 (2006) 991.

[4] M.J. Ramsey-Musolf and S. Su, Phys. Rept. 456 (2008) 1.

[5] J.D. Jackson, Phys. Rev. 106 (1957) 517.

[6] V. Gudkov, G. L. Greene and J. R. Calarco, Phys. Rev. C 73 (2006) 035501.

[7] W-M Yao, et al., J. Phys. G 33, 1 (2006).

[8] Nab experiment homepage: http://nab.phys.virginia.edu.

[9] S. Baeßler et al., to appear soon in Eur. Phys. J. (2008).

[10] F.E. Wietfeldt, et al., Nucl. Instr. and Meth. Phys. Res. A 545 (2005) 181.

[11] J.D. Bowman, J. Res. NIST 110 (2005) 407.

[12] http://geant4.web.cern.ch/geant4/,

[13] P. R. Huffman, et al., "Beamline Performance Simulations for the FnPB", private communication, 2005. Initial measurements performed in the FnPB up to the time of this writing are in agreement with the simulation.

[14] http://nab.phys.virginia.edu/nab_proposal.pdf. 